\title{
Correction to: Using feedback requests to actively involve assessees in peer assessment: effects on the assessor's feedback content and assessee's agreement with feedback
}

\author{
Michiel Voet $^{1}$ (D) Mario Gielen ${ }^{2} \cdot$ Ruth Boelens $^{1} \cdot$ \\ Bram De Wever ${ }^{1}$
}

Published online: 7 May 2018

(C) Instituto Superior de Psicologia Aplicada, Lisboa, Portugal and Springer Science+Business Media B.V., part of Springer Nature 2018

\section{Correction to: European Journal of Educational Psychology (2018) 33(1):145-164 https://doi.org/10.1007/s10212-017-0345-x}

Due to an error on the publisher's side, the authors' corrections to the proofs were unfortunately not incorporated into the original article. As a result, all references to the authors' own work remained blinded, and minor textual errors went unattended.

A link to the corrected version of the article can be found below, together with the full references to the authors' own work and the article corrections.

The online version of the original article can be found at https://doi.org/10.1007/s10212-017-0345-X

Michiel Voet

michiel.voet@ugent.be

Mario Gielen

mario.gielen@uhasselt.be

Ruth Boelens

ruth.boelens@ugent.be

Bram De Wever

bram.dewever@ugent.be

1 Department of Educational Studies, Ghent University, Henri Dunantlaan 2, 9000 Ghent, Belgium

2 School for Transportation Sciences, Hasselt University, Wetenschapspark 5, 3590 Diepenbeek, Belgium 


\section{Link to the corrected version of the article:}

http://www.tecolab.ugent.be/pubs/2018_Voet_Gielen_EJPE_Peer_Assessment.pdf

\section{Full references to author's own work:}

Authors (2015a)

Gielen, M. \& De Wever, B. (2015a). Scripting the role of assessor and assessee in peer assessment in a wiki environment: impact on feedback quality and product improvement. Computers \& Education, 88(1), 370-386.

Authors (2015b)

Gielen, M., \& De Wever, B. (2015b). Structuring peer assessment: comparing the impact of the degree of structure on peer feedback content. Computers in Human Behavior, 52(1),315325 .

Authors (2015c)

Gielen, M., \& De Wever, B. (2015c). Structuring the peer assessment process: a multilevel approach for the impact on product improvement and feedback quality. Journal of Computer Assisted Learning, 31(5), 435-449.

\section{Article corrections:}

Abstract:

Sentence found in lines 17-18 should be rewritten as: The use of a feedback request did, however, not have a significant effect on agreement with feedback.

Introduction:

Page 2, first paragraph, line 7 should be rewritten as: More specifically, research has shown that peer assessment draws on several higher order thinking skills, such as critical thinking, problem solving, and decision-making, and may therefore contribute to the development of these skills (King 2002).

Page 3, line 4 should be rewritten as: Regarding the question as to how usefulness of feedback can then be operationalized, the work by Strijbos et al. (2010) suggests that students' perceptions of feedback's usefulness are indistinguishable from their agreement with this feedback.

Research questions:

Page 3, lines 1 to 3 should be rewritten as: In short, the aim of the present study is to investigate how a more active involvement of the assessee in the feedback process, in the form of a feedback request, might influence the content of the assessor's feedback, as well as the assessee's agreement with the feedback.

Page 3, line 5 should be rewritten as: The effects of the feedback request are investigated in relation to those of an explication of assessment criteria for the assessor, which is commonly used by research that aims to support peer assessment (Authors 2015c; Falchikov 1995; Panadero and Jonsson 2013; Topping 1998).

Task:

Page 4, line 1 should be rewritten as: As part of an educational studies program, 125 firstyear students were divided over 27 groups, with three to five students per group, and given an assignment on writing a research abstract.

Page 5, figure 1, row 2 column 4 should be rewritten as: Saying that, I did change a few things: I have downsized the questions section to reduce the length of my abstract.

Data analysis:

Page 6, first paragraph, line 11 should be rewritten as: Due to one student failing to provide feedback during one of the cycles, 157 completed feedback templates were available for analysis. 
Page 8 , fourth paragraph, line 9 should be rewritten as: The interrater reliability for students' agreement with feedback was checked by a third coder, who independently coded 1166 segments of evaluations.

Page 8, fourth paragraph, line 11 should be rewritten as: Cohen's kappa for students' agreement with feedback (i.e., four categories: agreement, partial agreement, disagreement, no evaluation) was .92 , again indicating good interrater reliability.

Page 8, sixth paragraph, line 5 should be rewritten as: Model estimation then began with the estimation of two null models: one for feedback content and one for students' agreement with feedback.

The content of the assessor's feedback:

Page 8, first paragraph, line 1 should be rewritten as: To examine conditions' effect on the evolution of feedback content from cycle 1 to cycle 3, a multivariate multilevel growth curve model was estimated, with three dependent variables: verifications, informative elaborations, and suggestive elaborations.

Page 11, seventh paragraph, lines 6 to 7 should be rewritten as: As noted before, while these initial differences between conditions are taken into account by the growth model and therefore do not complicate the analysis, there is unfortunately no clear-cut explanation for these differences.

References:

Additional References:

Webb, N. M., \& Mastergeorge, A. (2003). Promoting effective helping behavior in peerdirected groups. International Journal of Educational Research, 39(1-2), 73-97.

Prins, F. J., \& Mainhard, M. T. (2009). Fostering students' self-regulation during feedback dialogues in vocational education. Paper presented at the 13th biennial EARLI conference, Amsterdam, The Netherlands. 